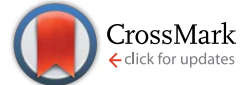

Cite this: RSC Adv., 2015, 5, 91619

Received 26th July 2015 Accepted 22nd October 2015

DOI: $10.1039 / c 5 r a 14829 d$

www.rsc.org/advances

\title{
A self-assembling polycationic nanocarrier that exhibits exceptional gene transfection efficiency $\dagger$
}

\begin{abstract}
Kishor Sarkar, ${ }^{a}$ Sai Rama Krishna Meka, ${ }^{\mathrm{b}}$ Giridhar Madras ${ }^{\mathrm{a}}$ and Kaushik Chatterjee ${ }^{\star b}$
The lack of an efficient and safe carrier is a major impediment in the field of gene therapy. Although gelatin (GT), a naturally derived polymer, is widely used in drug delivery applications, it is unable to bind DNA efficiently. In this study, a novel polycationic gene carrier was prepared by conjugation of low molecular weight polyethyleneimine (LPEI) with GT through 4-bromonaphthaleic anhydride as a coupling agent to avoid self crosslinking. Self-assembly of LPEI conjugated GT (GT-LPEI) with plasmid DNA (pDNA) yielded nanoparticles with high gene complexation ability to form $\sim 250 \mathrm{~nm}$ cylindrical nanoparticles with a zeta potential of $\sim 27 \mathrm{mV}$. GT-LPEI showed exceptionally high transfection efficiency $(>90 \%)$ in various mammalian cells including primary stem cells with minimal cytotoxicity. The transfection efficiency of GT-LPEI significantly surpassed that of many commercial reagents. The high gene transfection expression was confirmed in vivo. Thus, GT-LPEI is shown to be a promising nonviral carrier for potential use in gene therapy.
\end{abstract}

\section{Introduction}

Despite the development of several carriers in recent years, the lack of an efficient and safe carrier remains a key bottleneck in the field of gene therapy. ${ }^{1-3}$ Owing to several advantages such as low toxicity, nonimmunogenicity, versatility in chemical modification and low production cost, nonviral carriers have attracted tremendous attention over their viral counterparts in the field of gene therapy. ${ }^{4,5}$ Liposomes and cationic polymers such as polyethyleneimine (PEI), chitosan and dendrimers are the most widely used nonviral carriers but they exhibit low transfection efficiency with high toxicity. ${ }^{6-8}$ Among the cationic polymers, PEI is considered as the "gold standard" due to its unique advantages including substantial buffering capacity and high transfection efficiency. ${ }^{9}$ High molecular weight branched PEI (25 kDa) is the most widely used nonviral carrier due to its high transfection efficiency. ${ }^{10}$ But its severe toxicity limits its application. On the other hand, low molecular weight PEI (2 kDa) induces lower cytotoxicity but with significantly lower transfection efficiency. ${ }^{\mathbf{1 1}}$

Several extracellular barriers such as degradation by serum nucleases, nonspecific delivery and intracellular barriers including lysosomal degradation, cytosolic degradation and poor nuclear transport are the major limiting factors typically affecting nonviral gene transfection. ${ }^{\mathbf{1 2 , 1 3}}$ To overcome these

${ }^{a}$ Department of Chemical Engineering, Indian Institute of Science, Bangalore 560012, India

${ }^{b}$ Department of Materials Engineering, Indian Institute of Science, Bangalore 560012, India. E-mail: kchatterjee@materials.iisc.ernet.in; Tel: +91-80-2293-3408

$\dagger$ Electronic supplementary information (ESI) available. See DOI: 10.1039/c5ra14829d barriers, various strategies such as targeted delivery through ligand conjugation and improvement in cytosolic delivery using fusogenic peptides have been employed to enhance transfection efficiency. ${ }^{\mathbf{1 4 - 1 7}}$ However, the transfection efficiency of nonviral carriers is still much lower than the viral vectors.

Recently, natural polymers such as proteins, peptides and polysaccharides have been widely used for drug delivery applications due their inherent nontoxicity, biodegradability and biocompatibility. ${ }^{18-21}$ Among these, gelatin (GT) has a long history of successful use as an additive in pharmaceutics, food items and cosmetics. GT is approved as a GRAS (generally regarded as safe) material by the FDA (United States Food and Drug Administration). ${ }^{22}$ GT obtained from collagen through alkaline or acid hydrolysis is a denatured protein. ${ }^{23}$ GT possesses recognition sites such as the RGD (Arg-Gly-Asp) sequence to facilitate easy binding to cells. ${ }^{24}$ GT nanoparticles have been most widely used for drug delivery applications such as antimicrobial, ${ }^{25}$ anticancer, ${ }^{26}$ anti-diabetic, ${ }^{27}$ anti-inflammatory $^{28}$ and anti-HIV ${ }^{29}$ drugs among others. However, GT is less studied for gene delivery applications. In the last decade, Amiji and co-workers have reported on GT nanoparticle based gene delivery vehicles for systemic and oral gene therapy. ${ }^{30-32} \mathrm{~A}$ few other groups have also used modified GT for gene delivery. ${ }^{33,34}$

GT has low cationic charge and as a result it has low complexation capability with negatively charged DNA. Modification of GT with low molecular weight PEI (LPEI) offers a potential route to enhance its DNA complexation ability. LPEI is less cytotoxic than its higher molecular weight counterparts although its transfection efficiency is lower due to poor DNA complexation ability. Conjugation of GT to LPEI was envisaged 
to provide a minimally cytotoxic carrier with cell binding sites and good DNA complexation ability for high transfection efficiency. However, GT contains both carboxylic acid and primary amine groups. Hence, there is a strong tendency to selfcrosslink during cationic modification through conjugation of cationic polymers such as PEI using the conventional carbodiimide coupling reaction. It is envisaged that water soluble LPEI conjugated GT (GT-LPEI) can efficiently complex with pDNA by self-assembly into a compact nanoparticle as a vehicle for gene transfection. Thus, the objective of this study was to prepare GT-LPEI without crosslinking of GT and assess its potential for gene delivery.

Herein, we report on the synthesis of water soluble GT-LPEI using 4-bromonaphthaleic anhydride as the coupling agent. The reaction was a two stage process including the reaction between GT and 4-bromonaphthaleic anhydride to block the primary amine groups of GT followed by conjugation of LPEI through substitution reaction of the bromide groups by primary amine groups of LPEI. Self-assembled GT-LPEI/pDNA nanoparticles were prepared by complex coacervation method through electrostatic interactions between the positively charged GT-LPEI and the negatively charged pDNA. The transfection efficiency and cell viability of GT-LPEI were assessed in a variety of mammalian cells and confirmed in vivo. The cellular uptake mechanisms and intracellular distribution were also characterized.

\section{Materials and methods}

Type-B gelatin (225 bloom strength) with an isoelectric point of 4.7-5.2 and weight average molecular weight $\left(M_{\mathrm{w}}\right) 47.5 \mathrm{kDa}$, branched polyethyleneimine (1.8 and $25 \mathrm{kDa})$, 4-bromo-1,8naphthaleic anhydride, anhydrous dimethyl sulfoxide, 3-(4,5dimethylthiazol-2-yl)-2,5-diphenyltetrazolium bromide (MTT), ethidium bromide, chlorpromazine (CPZ), genistein (GEN), amiloride (AMIL), nocodazole (NOC), cytocalasin D (Cy-D) and 2,4,6-trinitrobenzene sulfonic acid (TNBS) were purchased from Sigma-Aldrich (USA). SnakeSkin cellulose acetate dialysis tubing (3.5 kDa MW cut off) was obtained from Thermo Scientific. Dulbecco's modified Eagle's medium (DMEM), knockout DMEM, alpha minimum essential medium ( $\alpha$-MEM), penicillin-streptomycin, trypsin and fetal bovine serum (FBS) were purchased from Invitrogen-Gibco (Carlsbad, Calif).

Plasmid DNA (pDNA) encoding for green fluorescence (LifeAct-TagGFP2) and luciferase gene (Luc ${ }^{+}$) (PGL3-Luc pDNA) under the control of the cytomegalovirus promoter/enhancer were obtained from Ibidi (San Diego, CA) and Promega (USA), respectively. D-Luciferin-sodium salt was obtained from BioVision. Vectors were propagated in competent Escherichia coli DH5 $\alpha$ cells. Ultrapure endotoxin free plasmid DNA was prepared using the QIAfilter Midi kit (Qiagen, Valencia, CA) according to the manufacturer's instructions. Plasmid DNA was diluted in ultrapure water (Invitrogen) to a final concentration of $1 \mathrm{mg} \mathrm{mL} \mathrm{m}^{-1}$ DNA. All other reagents were analytical grade and were used directly without further modification.

\subsection{Synthesis of PEI conjugated gelatin}

Low molecular weight PEI conjugated GT (GT-LPEI) was synthesized according to a previous report on preparation of PEI conjugated chitosan. ${ }^{35}$ The synthesis of GT-LPEI consisted of two consecutive steps (i) synthesis of $N$-(4-bromonaphthalimide)gelatin (Comp 3) followed by (ii) conjugation of LPEI with GT through a substitution reaction as shown in Fig. 1. Briefly, $1.0 \mathrm{~g}$ GT (Comp 1) was dissolved into $50 \mathrm{~mL}$ DMSO containing 4bromo-1,8-naphthalic anhydride (Comp 2) at different weight ratios as shown in Table 1 . The reaction mixture was refluxed at $80^{\circ} \mathrm{C}$ under nitrogen atmosphere for $3 \mathrm{~h}$. Thereafter, the reaction mixture was dialyzed using cellulose acetate dialysis membrane against double distilled water for 3 days to remove DMSO and unreacted Comp 2. Finally, the product was lyophilized for 3 days to get a brown colored Comp 3.

GT-LPEI was prepared by reaction between Comp 3 and excess amount of PEI aqueous solution as follows. $100 \mathrm{mg}$ of Comp 3 was dispersed in $50 \mathrm{~mL}$ aqueous solution of PEI $(10 \%$ PEI solution) and the reaction mixture was then refluxed at 80 ${ }^{\circ} \mathrm{C}$ under nitrogen atmosphere for $3 \mathrm{~h}$. The product was dialyzed against double distilled water for 3 days to remove unreacted PEI followed by lyophilization for 3 days to get a yellow colored final product GT-LPEI, Comp 4.

\subsection{Characterization}

The synthesis of GT-LPEI was characterized by Fourier transform infrared (FTIR) spectroscopy using ATR (attenuated total reflection) FTIR spectrometer (Perkin Elmer Spectrum 100 FTIR). All spectra were carried out within the frequency range of $4000-600 \mathrm{~cm}^{-1}$ for 42 consecutive scans. The proton nuclear magnetic resonance $\left({ }^{1} \mathrm{H}\right.$ NMR) spectra were determined on a Bruker AV 3000 Supercon NMR system (Germany) at 400 $\mathrm{MHz}$ using $\mathrm{D}_{2} \mathrm{O}$ and DMSO-d6 as solvent. Chemical shifts $(\delta)$ were reported in ppm using tetramethylsilane (TMS) as an internal reference. The molecular weight of GT-LPEI was determined by gel permeation chromatography (GPC) using $0.1 \mathrm{M}$ sodium chloride solution as eluent at flow rate of $0.3 \mathrm{~mL}$ $\min ^{-1}$ by Waters PC2 separation module and Waters 2414 refractive index detector. Tri-nitro benzene sulfonic acid (TNBS) assay was carried out for primary amine quantification of GT-LPEI. ${ }^{36}$

\subsection{Preparation of polymer/pDNA complexes}

Polymer/pDNA complexes were prepared by complex coacervation method according to a previous study. ${ }^{37}$ Gelatin and different GT-LPEI stock solutions dissolved in double distilled water $\left(\mathrm{ddH}_{2} \mathrm{O}\right)$ were diluted to $1 \mathrm{mg} \mathrm{mL} \mathrm{m}^{-1}$ concentration using $\mathrm{ddH}_{2} \mathrm{O}$. Independently, pDNA stock solution was diluted to 100 $\mu \mathrm{g} \mathrm{mL}^{-1}$ concentration in $25 \mathrm{mM}$ sodium sulfate solution. Thereafter, equal volumes $(50 \mu \mathrm{L})$ of polymer and pDNA solutions were mixed together at different weight ratios (polymer to pDNA weight ratios of $1: 1,5: 1,10: 1,15: 1,20: 1,25: 1$ and $30: 1$ ) and immediately vortexed for $20-30$ s followed by incubation at room temperature for $20 \mathrm{~min}$ to form the polymer/ pDNA complexes. 


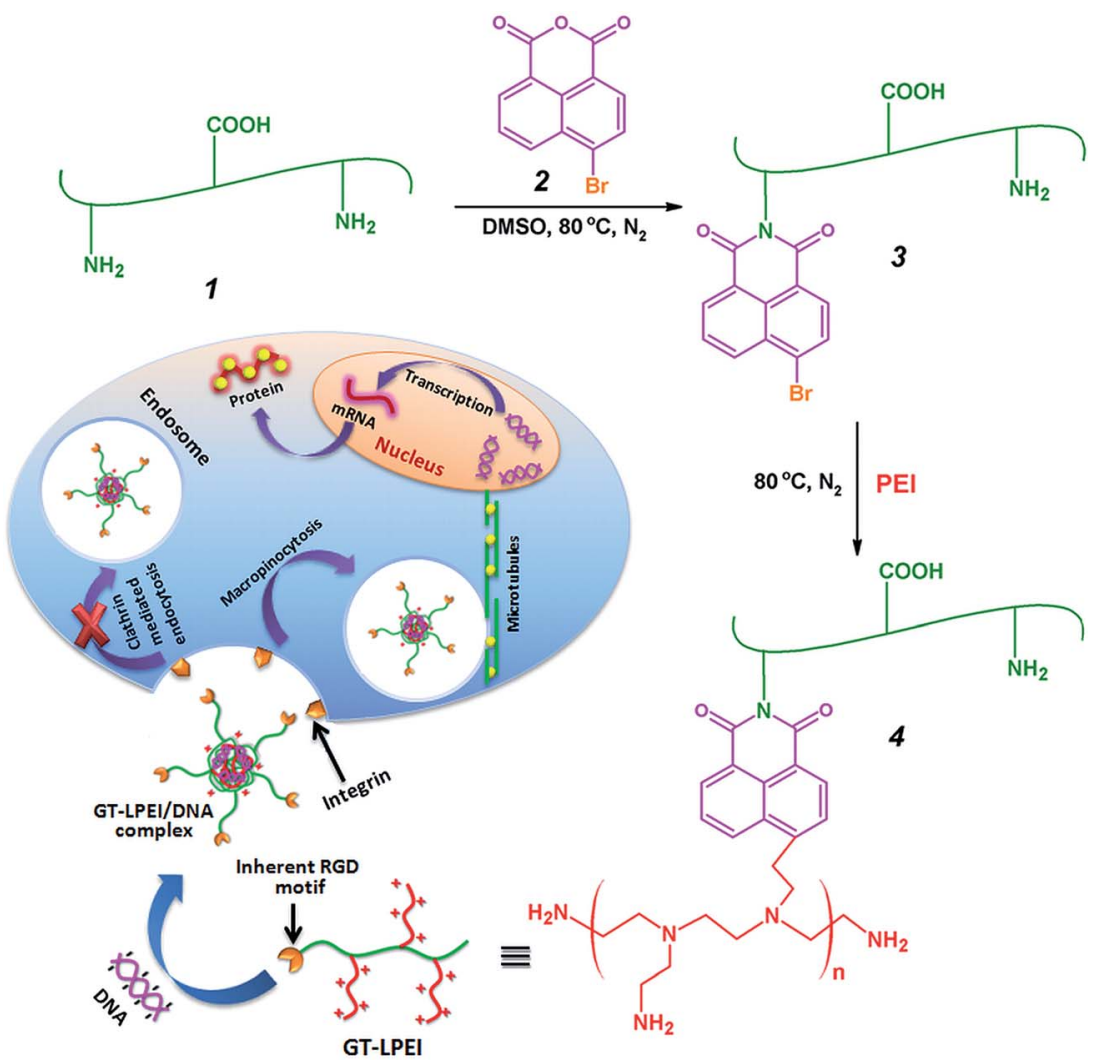

Fig. 1 Schematic diagram for synthesis of GT-LPEI and cellular uptake of GT-LPEI/pDNA complex.

\subsection{Characterization of polymer/pDNA complexes}

The complexation capability of GT and GT-LPEI with pDNA was characterized by the agarose gel electrophoresis assay. Polymer/ pDNA complexes at different weight ratios were freshly prepared as described above prior to the electrophoresis assay. Thereafter, polymer/pDNA complexes containing $0.5 \mu \mathrm{g}$ pDNA in each weight ratio were loaded in $0.8 \%$ agarose gel prepared in TAE buffer ( $40 \mathrm{mM}$ trisacetate and $1 \mathrm{mM}$ EDTA solution) containing ethidium bromide $\left(10 \mu \mathrm{g} \mathrm{mL}^{-1}\right)$ as DNA visualizer. The gel was run at $100 \mathrm{~V}$ for $40 \mathrm{~min}$ and subsequently the gel picture

Table 1 Chemical composition, primary amine content, zeta potential and molecular weight of GT-LPEI

\begin{tabular}{|c|c|c|c|c|c|c|}
\hline \multirow[b]{2}{*}{ Sample code } & \multicolumn{3}{|c|}{$\begin{array}{l}\text { Composition } \\
\text { (weight ratio) }\end{array}$} & \multirow[b]{2}{*}{$\% \mathrm{NH}_{2}{ }^{a}$} & \multirow{2}{*}{$\begin{array}{l}\text { Zeta } \\
\text { potential } \\
(\mathrm{mV})\end{array}$} & \multirow{2}{*}{$\begin{array}{l}\text { Molecular } \\
\text { weight }\left(M_{\mathrm{w}}\right) \\
(\mathrm{kDa})\end{array}$} \\
\hline & GT & $\begin{array}{l}\text { Comp } \\
2\end{array}$ & LPEI & & & \\
\hline GT-LPEI-0 & 1 & 0.00 & 0 & $14.0 \pm 3.5$ & $2.4 \pm 1.9$ & 47.5 \\
\hline GT-LPEI-0.01 & 1 & 0.01 & 50 & $17.2 \pm 1.6$ & $5.2 \pm 2.6$ & 48.3 \\
\hline GT-LPEI-0.06 & 1 & 0.06 & 50 & $23.4 \pm 2.3$ & $11.9 \pm 4.2$ & 50.3 \\
\hline GT-LPEI-0.12 & 1 & 0.12 & 50 & $31.0 \pm 3.2$ & $18.5 \pm 5.1$ & 55.2 \\
\hline GT-LPEI-0.18 & 1 & 0.18 & 50 & $36.7 \pm 2.7$ & $26.4 \pm 3.5$ & 56.4 \\
\hline GT-LPEI-0.20 & 1 & 0.20 & 50 & $39.1 \pm 1.8$ & $36.5 \pm 2.8$ & 59.7 \\
\hline
\end{tabular}

${ }^{a}$ Determined by TNBS assay. was captured using myECL Imager gel documentation (Thermo Scientific).

The DNA complexation capability of GT and GT-LPEI was further confirmed by ethidium bromide (EtBr) assay according to a previous study. ${ }^{38}$ Briefly, pDNA/EtBr complex was prepared at a molar ratio of 10:1 (pDNA : EtBr molar ratio). Equal volume of GT and GT-LPEI solution with respect to pDNA/EtBr complex were added to the pDNA/EtBr complex at different weight ratio $(1: 1,5: 1,10: 1,15: 1,20: 1,25: 1$ and $30: 1)$ to the pDNA and incubated for $30 \mathrm{~min}$ at $25{ }^{\circ} \mathrm{C}$. Subsequently, the fluorescence intensity with excitation of $510 \mathrm{~nm}$ and emission of $605 \mathrm{~nm}$ was measured using a microplate reader (Synergy HT, Biotek).

The particle size and zeta potential of GT/pDNA and GTLPEI/pDNA complexes at different weight ratios were measured by dynamic light scattering (DLS) using Zetasizer Nano ZS (Malvern Instrument, UK) at $37{ }^{\circ} \mathrm{C}$. Before measuring the particle size and zeta potential, the polymer/pDNA complexes were freshly prepared at different weight ratios and diluted to $1.0 \mathrm{~mL}$ with filtered $\mathrm{dd}_{2} \mathrm{O}$. For each experiment, three samples were run. The effect of medium (DMEM) and serum containing medium (10\% FBS supplemented DMEM) on particle size and zeta potential were also measured.

The particle size and morphology of GT/pDNA and GT-LPEI/ pDNA complexes were further observed by atomic force microscopy (AFM, Park systems NX-10) and the images were analyzed by XEI AFM image processing software. Freshly 
prepared $5 \mu \mathrm{L}$ of polymer/pDNA complex diluted in $\mathrm{dd}_{2} \mathrm{O}$ was deposited on freshly cleaved mica foil and dried at room temperature before imaging. The particles were also characterized by transmission electron microscopy (TEM; JEOL, Model-1011) at an accelerating voltage of $80 \mathrm{kV}$.

\subsection{Cell culture}

HeLa (human cervical cancer cells), A549 (human lung adenocarcinoma cells), SVEC (mouse microvascular endothelial cells), MC3T3-E1 (mouse osteoblasts), RAW 264.7 (murine macrophages) and human mesenchymal stem cells (hMSCs) were used for transfection and cytotoxicity studies in vitro. All cell lines were obtained from ATCC. Primary bone marrow derived hMSCs from a 25 year old male donor were obtained from Stempeutics, India. HeLa, A549, SVEC and RAW 264.7 cells were cultured in DMEM medium containing 10\% (v/v) FBS, 1\% penicillin-streptomycin and $1 \%$ of $2 \mathrm{mM}$-glutamine in $5 \%$ $\mathrm{CO}_{2}$ and $37^{\circ} \mathrm{C}$. MC3T3-E1 cells and hMSCs were cultured in $\alpha$ MEM and knockout DMEM, respectively, containing 10\% serum and antibiotics as above.

\subsection{In vitro cytotoxicity assay}

The toxicity of GT and GT-LPEI at different concentrations ( 0 , 10, 20, 50, 100, 200, 300 and $500 \mu \mathrm{g} \mathrm{mL}^{-1}$ ) was optimized in HeLa cells. Branched PEIs ( 2 and $25 \mathrm{kDa}$ ) were used as positive controls. $5 \times 10^{3}$ cells suspended in complete culture medium were seeded in each well of a 96-well plate and cultured for $24 \mathrm{~h}$. The polymer solutions at different concentrations were added to the cells and incubated in the $\mathrm{CO}_{2}$ incubator for $24 \mathrm{~h} .5 .0 \mu \mathrm{L}$ of MTT solution (5 mg mL $\mathrm{mL}^{-1}$ ) diluted to $100 \mu \mathrm{L}$ in DMEM medium was added to the treated cell and incubated for $4 \mathrm{~h}$. The MTT solution was replaced by $100 \mu \mathrm{L}$ DMSO to dissolve the formazan crystals. The absorbance of the solution was measured at $570 \mathrm{~nm}$ using a microplate reader (Synergy HT, BioTek instrument) to calculate the cell viability (\%) as follows:

$$
\text { Cell viability }(\%)=\frac{\mathrm{OD}_{570(\text { sample })}}{\mathrm{OD}_{570(\text { control })}} \times 100
$$

where, $\mathrm{OD}_{570 \text { (sample) }}$ and $\mathrm{OD}_{570 \text { (control) }}$ were the absorbance values of the polymer treated cells and untreated cells, respectively. Three replicates were used for each measurement.

The toxicity of GT/pDNA and GT-LPEI-0.20/pDNA complexes at different weight ratios such as $1: 1,5: 1,10: 1,15: 1,20: 1$, $25: 1$ and $30: 1$ was also measured on different cells such as HeLa, A549, SVEC, MC3T3-E1, RAW 264.7 and hMSCs. Freshly prepared polymer/pDNA complexes were added to the cells and incubated for $4 \mathrm{~h}$ in $\mathrm{CO}_{2}$ incubator at $37^{\circ} \mathrm{C}$. The polymer/pDNA complex containing solution was replaced by fresh growth medium containing serum and further incubated for $44 \mathrm{~h}$. Lipofectamine 2000 (LF 2000)/pDNA (Life Technologies) and PEI ( $25 \mathrm{kDa}) /$ pDNA complexes were used as positive controls. The cell viability (\%) was measured as described above.

\subsection{In vitro transfection study}

The transfection efficiency of different GT-LPEIs was optimized in HeLa cells. HeLa cells were seeded in a 24 well plate at a density of $5 \times 10^{4}$ cells per well and cultured until $70-80 \%$ confluency. GT/pDNA and GT-LPEI/pDNA complexes were freshly prepared at different weight ratios $(1: 1,5: 1,10: 1$, $15: 1,20: 1,25: 1$ and $30: 1$ ) as described above containing $1 \mu \mathrm{g}$ pDNA for each weight ratio. The polymer/pDNA complexes diluted with complete culture medium were added to the cell and incubated for $4 \mathrm{~h}$ in $\mathrm{CO}_{2}$ incubator at $37{ }^{\circ} \mathrm{C}$. The transfection medium was replaced by fresh complete culture medium and further incubated for $44 \mathrm{~h}$. pDNA alone and LF 2000/pDNA were used as negative and positive controls, respectively. Branched PEI (25 kDa)/pDNA complex at N/P ratio (nitrogen to phosphate ratio) of 10 was also used as a positive control. The green fluorescence expression of the transfected cells was observed by fluorescence microscopy (Olympus IX53, Japan). Fluorescence-activated cell sorting (FACS, BectonDickinson) was used to quantify the transfection efficiency. The transfection efficiency of GT-LPEI-0.20 was further compared with other commercialized transfection reagents such as Lipofectamine 3000 (LF 3000, Life Technologies), DreamFect Gold (DF Gold, OZ Biosciences), TransIT 2020 and TransIT X2 (both Mirus Bio LLC). The effect of serum content on transfection efficiency was also observed.

The transfection efficiency of the carrier was further assessed in different cells such as A549, SVEC, MC3T3-E1 and RAW 264.7 cells and hMSCs. GT-LPEI-0.20/pDNA complexes were freshly prepared at three different weight ratios $(20: 1,25: 1$ and $30: 1)$ and the transfection study performed as above.

\subsection{Intracellular distribution and kinetics}

To understand the pathways involved in cellular uptake, different endocytic inhibitor drugs such as chlorpromazine $\left(10 \mu \mathrm{g} \mathrm{mL}^{-1}\right)$, genistein $(200 \mu \mathrm{M})$ and amiloride $(100 \mu \mathrm{M})$ were used to block clathrin, caveolae and macropinocytosis mediated endocytosis pathways, respectively. Nocodazole $(10 \mu \mathrm{M})$, cytochalasin D $(20 \mu \mathrm{M})$ and RGDS (200 $\mathrm{nM})$ were also used to block cellular cytoskeleton, microtubule and surface integrin, respectively. Low temperature $\left(4^{\circ} \mathrm{C}\right)$ was applied to block the energy-dependent endocytosis. Cells were treated with the different drugs for $1 \mathrm{~h}$ prior to the transfection. Thereafter, GTLPEI-0.20/pDNA complex at weight ratio of $25: 1$ was added to the drug treated cells in the presence of drugs and incubated for $4 \mathrm{~h}$. The media was replaced by fresh growth media and further incubated for another $20 \mathrm{~h}$. The transfection of untreated cell with GT-LPEI-0.20/pDNA complex was considered as control. The transfection efficiency was quantified by FACS analysis.

In order to track the intracellular distribution of GT-LPEI/ DNA complex after cellular uptake, pDNA was fluorescently labeled with Cy3 using Label IT nucleic acid labeling kit (Mirus Bio, USA). HeLa cells were seeded at a density of $1 \times 10^{5}$ cells per well in a 4-well chambered glass slide (SPL Life Sciences) and cultured for $24 \mathrm{~h}$. GT-LPEI-0.20/DNA complex at weight ratio of 25 : 1 containing $1 \mu \mathrm{g}$ Cy3-labeled pDNA was added to the cell and incubated for different time points such as 0, 0.5, 2 and $4 \mathrm{~h}$. At each time point, the cells were fixed with $3.7 \%(\mathrm{w} / \mathrm{v})$ formaldehyde for $30 \mathrm{~min}$ and stained with diamidino phenylindole (DAPI) for $10 \mathrm{~min}$. The intracellular distribution of the 
complexes at different time intervals was observed by confocal laser scanning microscopy (CLSM; Leica TCS Sp5). The intracellular distribution of GT-LPEI-0.20/DNA complex was compared with pDNA alone, PEI $(25 \mathrm{kDa}) / \mathrm{pDNA}$ complex at N/P ratio of 10 and LF 2000/pDNA complex.

\subsection{Lysotracker colocalization assay}

GT-LPEI-0.20 and PEI (25 kDa) were labeled with fluorescein isothiocyanate (FITC) according to the previous report. ${ }^{39} 1 \times 10^{5}$ HeLa cells were seeded in a 24 well plate and cultured for $24 \mathrm{~h}$. FITC labeled GT-LPEI-0.20/pDNA and PEI (25 kDa)/pDNA complexes at weight ratio of $25: 1$ and N/P ratio of 10 , respectively were prepared prior to the experiment. Polymer/DNA complexes were added to the cells in serum free medium and incubated at $37{ }^{\circ} \mathrm{C}$. After $4 \mathrm{~h}$, the medium containing the polymer/DNA complex was removed and washed thrice with fresh PBS. Lysotracker Red DND 99 (Life Technologies) diluted in serum free medium at concentration of $500 \mathrm{nM}$ was added to the cells followed by incubation for $1 \mathrm{~h}$ at $37^{\circ} \mathrm{C}$. Cells were washed three times after removing the medium and subsequently fixed using $4 \%$ paraformaldehyde solution followed by counter staining of nuclei by DAPI, and imaged using the CLSM.

\subsection{In vivo transfection}

To assess transfection in vivo, 6-8 week old, 20-25 $\mathrm{g}$ female $\mathrm{Balb} / \mathrm{c}$ mice were used and the animals were maintained in pathogen free environment at controlled temperature. The animal study was carried out by following the guidelines approved by Institutional Animal Ethics Committee of the Indian Institute of Science. PGL3-Luc pDNA was used for in vivo transfection. $100 \mu \mathrm{L}$ freshly prepared GT-LPEI-0.20/pDNA complex at weight ratio of $25: 1$ containing $40 \mu \mathrm{g}$ pDNA was injected intraperitoneally. pDNA alone and PEI (25 kDa)/pDNA complex at $\mathrm{N} / \mathrm{P}$ ratio of 10.0 were used as the negative and positive controls, respectively. Three animals $(n=3)$ were used in each group. The animals were injected intraperitoneally with $200 \mu \mathrm{L}$ of D-luciferin $\left(15 \mathrm{mg} \mathrm{mL} \mathrm{mL}^{-1}\right) 24 \mathrm{~h}$ post injection and anesthetized by isoflurane gas $(300 \mathrm{~mL}$ air flow with $3 \%$ isoflurane). The signal of luciferase gene expression was visualized by using bioluminescence IVIS imaging system (Caliper Life Science) after 5 min exposure under continuous supply of isoflurane gas ( $200 \mathrm{~mL}$ air with $2 \%$ isoflurane) and the images were captured using the Living Image software.

\subsection{Statistical analysis}

All the data are shown as the average \pm the standard deviation and one-way ANOVA was used to determine statistically significant differences ( $p$ values less than 0.05 ).

\section{Results and discussion}

\subsection{Synthesis and characterization of GT-LPEI}

GT-LPEI was synthesized by conjugation of low molecular weight PEI (1.8 kDa) with gelatin through naphthalimide moiety as shown in Fig. 1. Gelatin contains both primary amine and carboxylic acid groups. During the conjugation of PEI with gelatin through reaction of the primary amine groups of PEI and the carboxylic acid groups of gelatin by conventional carbodiimide chemistry, there is enhanced tendency to form crosslinked products through reaction of intramolecular primary amine and carboxylic acid groups of gelatin. This may reduce the DNA complexation ability and cellular binding capacity of the modified gelatin. To minimize the formation of crosslinked nanoparticles, the primary amine groups of gelatin were first blocked through reaction with 4-bromonaphthalic anhydride to form Comp 3. Subsequently, PEI was conjugated with Comp 3 through substitution reaction of the primary amine group of PEI and the bromide group of Comp 3 to synthesize GT-LPEI. A library of Comp 3 was synthesized by varying the weight ratios of GT and Comp 2 as shown in Table 1. Excess amount of PEI was reacted with different types of Comp 3 to synthesize different GT-LPEIs.

The synthesis of GT-LPEI was characterized by FTIR as shown in Fig. S1. $\dagger$ The FTIR spectra of GT showed a broad peak at $3446 \mathrm{~cm}^{-1}$ corresponding to the overlapped hydrogen bonded $\mathrm{O}-\mathrm{H}$ (hydroxyl group) stretching with $\mathrm{N}-\mathrm{H}$ stretching of the primary amine group in GT. The strong absorption peak at $1655 \mathrm{~cm}^{-1}$ for $\mathrm{C}=\mathrm{O}$ stretching of amide group indicates the presence of amide linkages in GT. After reaction of GT with Comp 2, two new peaks appeared at 1784 and $1732 \mathrm{~cm}^{-1}$ due to the symmetric and antisymmetric $\mathrm{C}=\mathrm{O}$ stretching of the naphthalimide group, respectively, confirming the synthesis of Comp 3. In addition, the peak for $\mathrm{O}-\mathrm{H}$ stretching shifted from $3446 \mathrm{~cm}^{-1}$ to $3420 \mathrm{~cm}^{-1}$ and became narrower, which indicates that the primary amine groups of GT reacted with naphthalic anhydride. The $\mathrm{O}-\mathrm{H}$ stretching peak shifted further to a higher value and became broader after conjugation of PEI with Comp 3 due to the overlap of hydrogen bonded O-H stretching with the $\mathrm{N}-\mathrm{H}$ stretching of primary amine groups in PEI. The strong absorption peak at $1567 \mathrm{~cm}^{-1}$ due to the $\mathrm{N}-\mathrm{H}$ stretching of primary amine groups confirms the conjugation of PEI with GT through the naphthalimide moiety.

The synthesis of GT-LPEI was further validated by ${ }^{1} \mathrm{H}$ NMR spectrometer. The ${ }^{1} \mathrm{H}$ NMR spectra of GT, Comp 3 and GT-LPEI0.20 are shown in Fig. S2. $\dagger$ The typical ${ }^{1} \mathrm{H}$ NMR spectra of GT (Fig. S2a $\dagger$ ) indicates the presence of different amino acid moieties in GT such as valine (Val), leucine (Leu) and isoleucine (Ile) (0.7-0.95 ppm); alanine (Ala) (1.3 ppm); arginine (Arg) (1.6 and $3.1 \mathrm{ppm}$ ) and proline (Pro) (3.55 ppm). ${ }^{40}$ A new broad multiplicity peak appeared at 7.6-8.8 ppm due to the $\mathrm{H}_{\alpha}$ and $\mathrm{H}_{\beta}$ protons present in the aromatic ring of the naphthalimide moiety indicating the synthesis of Comp 3 through reaction of GT and Comp 2 (Fig. S2b $†$ ). After conjugation of PEI with Comp 3 through substitution reaction of the bromide group of naphthalimide moiety by the primary amine group of PEI, a broad peak with multiplicity appeared at 2.3-3.0 ppm due to $-\mathrm{CH}_{2}-$ protons present in PEI confirming the synthesis of GTLPEI (Fig. S2c $\dagger$ ).

From Table 1, it is observed that the molecular weight of GT increased with the increase in the weight ratios of GT and Comp 2 resulting from the conjugation of LPEI with GT through the naphthalimide moiety. GT is insoluble in water at ambient temperature $\left(23^{\circ} \mathrm{C}\right)$ but is soluble in hot water. After reaction of 
GT with Comp 2, the solubility of GT decreased gradually with increasing weight ratio of GT : Comp 2 (as shown in Table 1) likely due to the increase in the content of hydrophobic aromatic moiety in the product Comp 3. However, all GT derivatives became water soluble at room temperature after conjugation of low molecular weight PEI with Comp 3. During reaction of Comp 3 and PEI, we used excess amount PEI (50 fold excess) to minimize intermolecular crosslinking. When lower amounts of PEI were used, the reaction of one molecule of PEI with two molecule of Comp 3 is more likely yielding water insoluble crosslinked products. In the presence of excess PEI, smaller sized PEI molecule can react preferentially with Comp 3 compared to the larger molecule (Comp 3). Similar phenomenon was observed in a previous study where water soluble chitosan was prepared by conjugation of polyamidoamine dendrimer with chitosan through maleic anhydride. ${ }^{41}$

\subsection{Preparation and characterization of polymer/DNA complexes}

A cationic polymer can form complex with negatively charged pDNA through electrostatic interactions. The complexation capability of GT and GT-LPEI was observed by agarose gel electrophoresis assay as shown in Fig. S3. $\dagger$ In agarose gel electrophoresis assay, GT/pDNA and GT-LPEI/PDNA complexes were prepared at different weight ratios $(1: 1,5: 1,10: 1,15: 1$, $20: 1,25: 1$ and $30: 1)$ containing $0.5 \mu \mathrm{g}$ of pDNA in each. From Fig. S3a, $\dagger$ it is seen that GT was not able to complex with all pDNA at any weight ratio due to its low cationic charge or zeta potential (Table 1). The DNA complexation capability of GT significantly increased after conjugation of cationic branched PEI with GT through the naphthalimide moiety. The complexation capability of GT-LPEI also increased with increase in amine content as shown in Table 1 (determined by TNBS assay). All GT-LPEIs of weight ratio of $5: 1$ and higher were able to complex all of the pDNA.

The DNA complexation capability of GT and GT-LPEI was further observed by EtBr assay as shown in Fig. S4. $\dagger$ In this assay, EtBr molecules intercalate in the base pair of double helix of pDNA resulting in strong fluorescence. As it is a reversible reaction, the addition of the cationic compound can displace the $\mathrm{EtBr}$ molecule lowering the fluorescence signal. From Fig. $\mathrm{S} 4, \dagger$ it can be seen that the relative fluorescence intensity of pDNA/EtBr complex decreased upon addition of GT and GTLPEI. Due to low cationic charge of GT, the change in relative fluorescence intensity was low. But GT-LPEI sharply decreased the fluorescence intensity due to the large positive charge as shown in Table 1 . The fluorescence intensity gradually decreased with the increase in polymer/DNA weight ratio. Among all the different GT-LPEIs, GT-LPEI-0.20 was most efficient due to its highest cationic character (Table 1). Results of the EtBr assay result corroborate the data from the agarose electrophoresis.

The particle size and zeta potential of polymer/DNA complex are important parameters for efficient cellular uptake. ${ }^{42}$ The particle size and zeta potential of GT/pDNA and GT-LPEI/pDNA complexes were determined by DLS. The average particle size of
GT/pDNA and GT-LPEI/pDNA complexes is shown in Fig. 2. Due to inefficient binding capability of GT with pDNA, the GT/pDNA complexes were large and the smallest particle size of GT/pDNA complex was $\sim 1000 \mathrm{~nm}$ at weight ratio of $30: 1$. But the particle size decreased significantly after conjugation of PEI with GT. GT-LPEI-0.20 formed the smallest particle among the all GTLPEIs due to its highest amine content and consequently higher zeta potential. The particle size was larger at low weight ratio but it decreased with increase in the weight ratio. GT-LPEI-0.20 formed smallest particle $(\sim 250 \mathrm{~nm})$ with pDNA at weight ratio of $25: 1$ but the particle size marginally increased with further increase of the weight ratio putatively due to the repulsion of the excessive positive charge offered by GT-LPEI.

Net positive charge of a polymer/DNA complex facilitates cellular uptake through electrostatic interactions between the positively charged complex and the negatively charged cellular membrane. Fig. 2b presents the measured zeta potential of GT/ pDNA and GT-LPEI/pDNA complexes at different weight ratios. It can be seen that the zeta potential of GT/pDNA complexes is negative up to weight ratio of $20: 1$ indicating that GT was not able to neutralize all the negative charges of pDNA because of its low cationic property. In contrast, GT-LPEI/pDNA complexes except GT-LPEI-0.01/pDNA and GT-LPEI-0.06/pDNA complex show positive zeta potential at all weight ratios. The zeta potential of GT-LPEI/pDNA complex increased with the increase in weight ratio. GT-LPEI-0.20/pDNA complex showed highest zeta potential among the all GT-LPEI/pDNA complexes because of its large cationic character. The zeta potential of GTLPEI-0.20/pDNA complex at weight ratio of $20: 1$ was $19.5 \pm 2.1$ $\mathrm{mV}$ and did not change markedly at higher ratios. Similar trends in zeta potential were observed in previous studies. ${ }^{\mathbf{4 3 - 4 5}}$ High zeta potential is helpful to stabilize the particle size through electrostatic repulsion. Low zeta potential of GT/pDNA complex may result in formation of large agglomerated particles compared to GT-LPEI/pDNA complexes which possess higher zeta potential.

In addition to the particle size and zeta potential of polymer/ DNA complex, the morphology of polymer/DNA complex is also a determining factor for efficient cellular uptake. ${ }^{46}$ The morphology of GT-LPEI-0.20/pDNA complex at weight ratio of 25 : 1 was observed by AFM as shown in Fig. $2 \mathrm{c}$ and d. The GTLPEI-0.20/pDNA complex was cylindrical having length of 128 $\mathrm{nm}$ and diameter of $53 \mathrm{~nm}$ (L/D ratio, aspect ratio of 2.4). TEM images of GT-LPEI-0.20/pDNA complex at weight ratio of $25: 1$ also showed the cylindrical morphology as shown in Fig. 2e and f. Some studies indicate that cylindrical morphology can facilitate in uptake leading to better transfection efficiency although such as shape dependence is not universal and depends on the carrier. A previous study showed that the particles having cylindrical morphology with aspect ratio of 3 (length $150 \mathrm{~nm}$ and diameter $50 \mathrm{~nm}$ ) showed better cellular uptake compared to spherical particles with aspect ratio 1 (length and diameter $200 \mathrm{~nm}) .{ }^{47}$ Another study also reported improved transfection in vivo with elongated particles. ${ }^{48}$ 


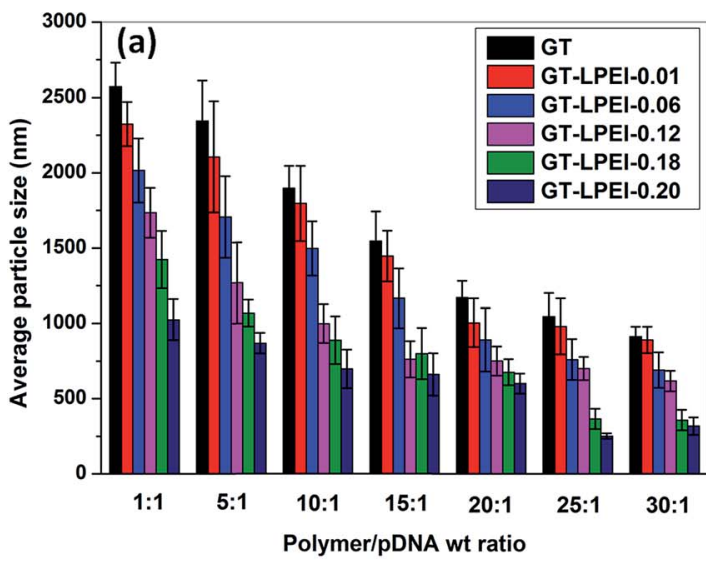

(c)
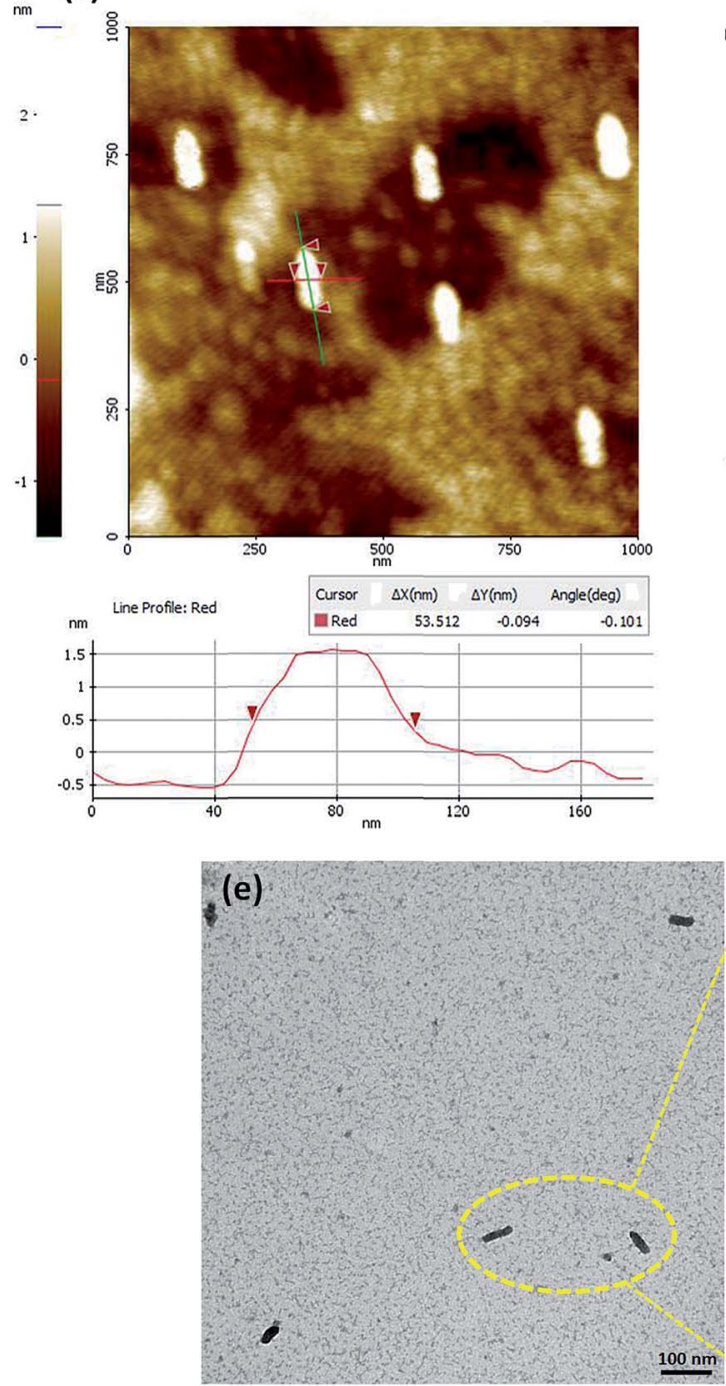

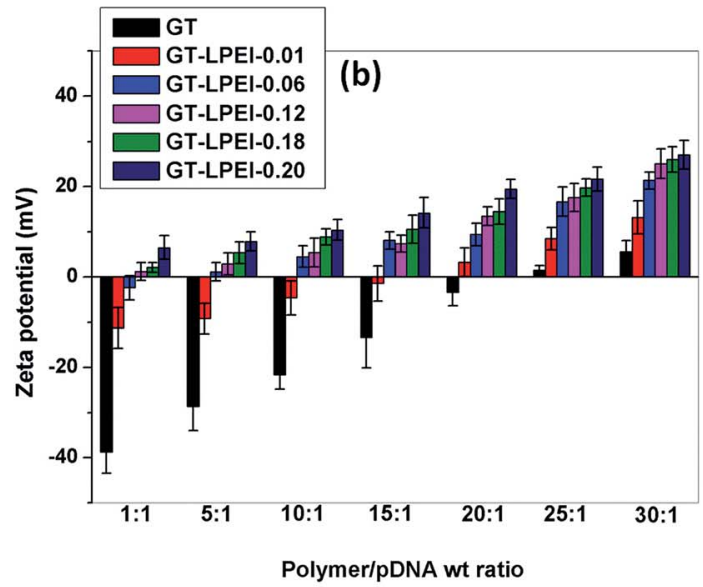

(d)
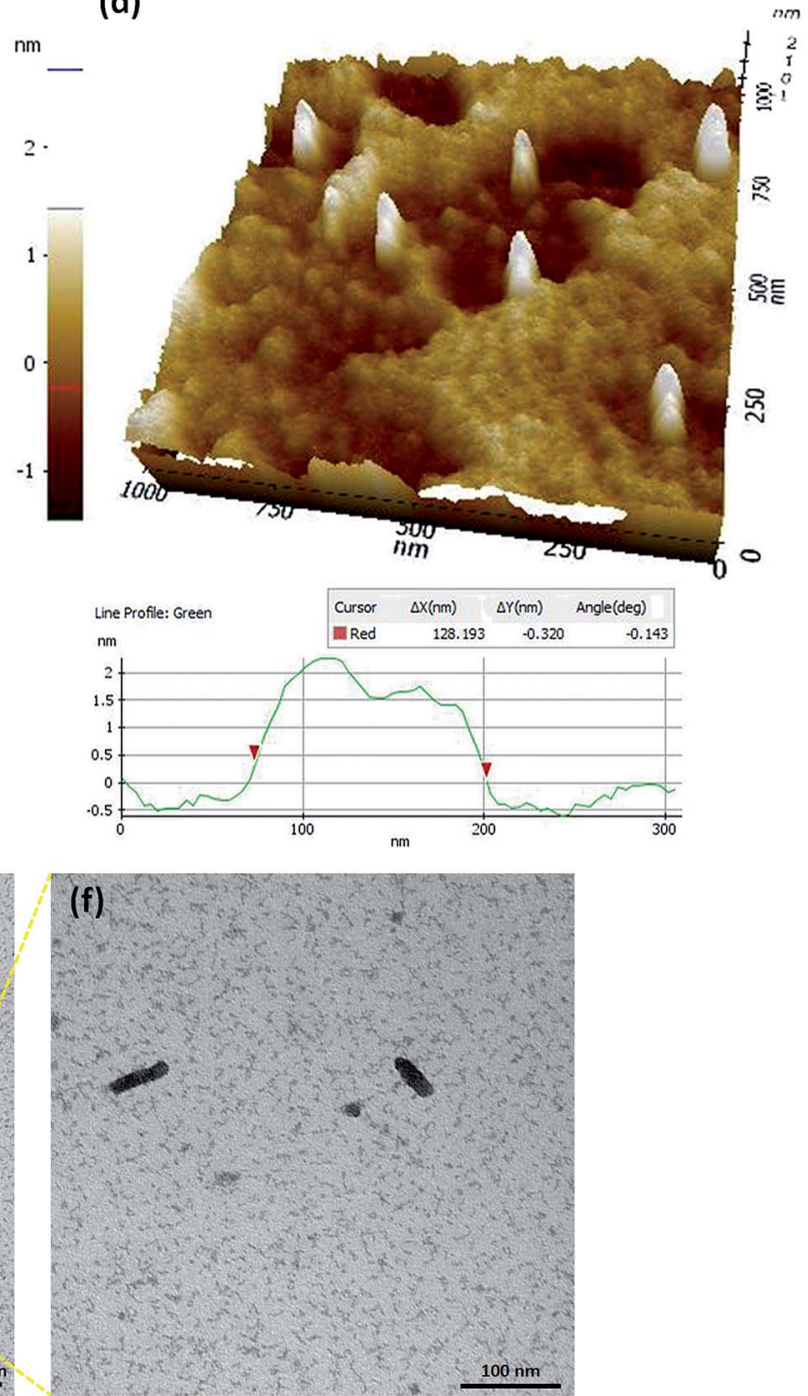

Fig. 2 (a) Average particle size and (b) zeta potential of GT/pDNA and GT-LPEIs/pDNA complexes at different weight ratios of $1: 1,5: 1,10: 1$, $15: 1,20: 1,25: 1$ and $30: 1$ determined by DLS. AFM images (c) height image and (d) 3D topography images of GT-LPEI-0.20/pDNA complex at weight ratio of $25: 1$. TEM micrographs of GT-LPEI-0.20/pDNA complex at weight ratio of $25: 1$ at low magnification (e) and high magnification (f). 


\subsection{In vitro cytotoxicity}

Low toxicity along with efficient transfection efficiency of the gene carrier system is a prime requirement for gene therapy applications in vivo. The toxicity of the modified gelatin at different concentrations $(0,10,20,50,100,200,300$ and $500 \mu \mathrm{g}$ $\mathrm{mL}^{-1}$ ) was evaluated in HeLa cells by MTT assay as shown in Fig. S5. $\dagger$ Not surprisingly, GT showed no toxicity in HeLa cell even at high concentration $\left(500 \mu \mathrm{g} \mathrm{mL}^{-1}\right)$ as also reported previously. ${ }^{49}$ However, the cell viability decreased after conjugation of PEI with GT although the toxicity of all GT-LPEIs were significantly lower compared to both high (25 kDa) and low (2 kDa) molecular weight PEI. The toxicity of all GT-LPEIs increased with increase in polymer concentration. GT-LPEI0.20 showed higher toxicity among the all GT-LPEIs likely due to its highest cationic property. Toxicity of cationic polymers is attributed to the destabilization of the cellular membrane through electrostatic interactions between negatively charged cellular membrane and the positively charged polymer. ${ }^{50}$ However, GT-LPEI-0.20 showed $50 \%$ cell viability at very high concentration of $320 \mu \mathrm{g} \mathrm{mL} \mathrm{m}^{-1}$, which was also significantly higher than that of both PEIs.

The cell viability of polymer/pDNA complexes at different weight ratios $(1: 1,5: 1,10: 1,15: 1,20: 1,25: 1$ and $30: 1)$ was also observed in various cells such as HeLa (Fig. S6a $\dagger$ ), A549 (Fig. S6b $\dagger$ ), SVEC (Fig. S6c $\dagger$ ), MC3T3-E1 (Fig. S6d †), RAW 264.7 (Fig. S6e $\dagger$ ) and hMSCs (Fig. S6f $\dagger$ ). Cell viability of GT-LPEI-0.20/ pDNA complexes at all weight ratios was $80 \%$ or better in all cells. Note that viability appears significantly improved in contrast to that of the uncomplexed polycation (Fig. S5†). The zeta potential remains high for the uncomplexed polycationic carrier but it becomes lower after complexation with the negatively charged DNA through charge neutralization. Bare GTLPEI-0.20 possesses high zeta potential value of $67.7 \pm 3.2 \mathrm{mV}$ but the value is lower $(27.4 \pm 2.5 \mathrm{mV})$ after complexation with pDNA at $30: 1$. Moreover, the actual concentration of polymer in polymer/pDNA complex used was less than $100 \mu \mathrm{g} \mathrm{mL} \mathrm{L}^{-1}$ of the polymer which yielded good transfection as discussed below. The toxicity of GT-LPEI-0.20/pDNA complexes increased
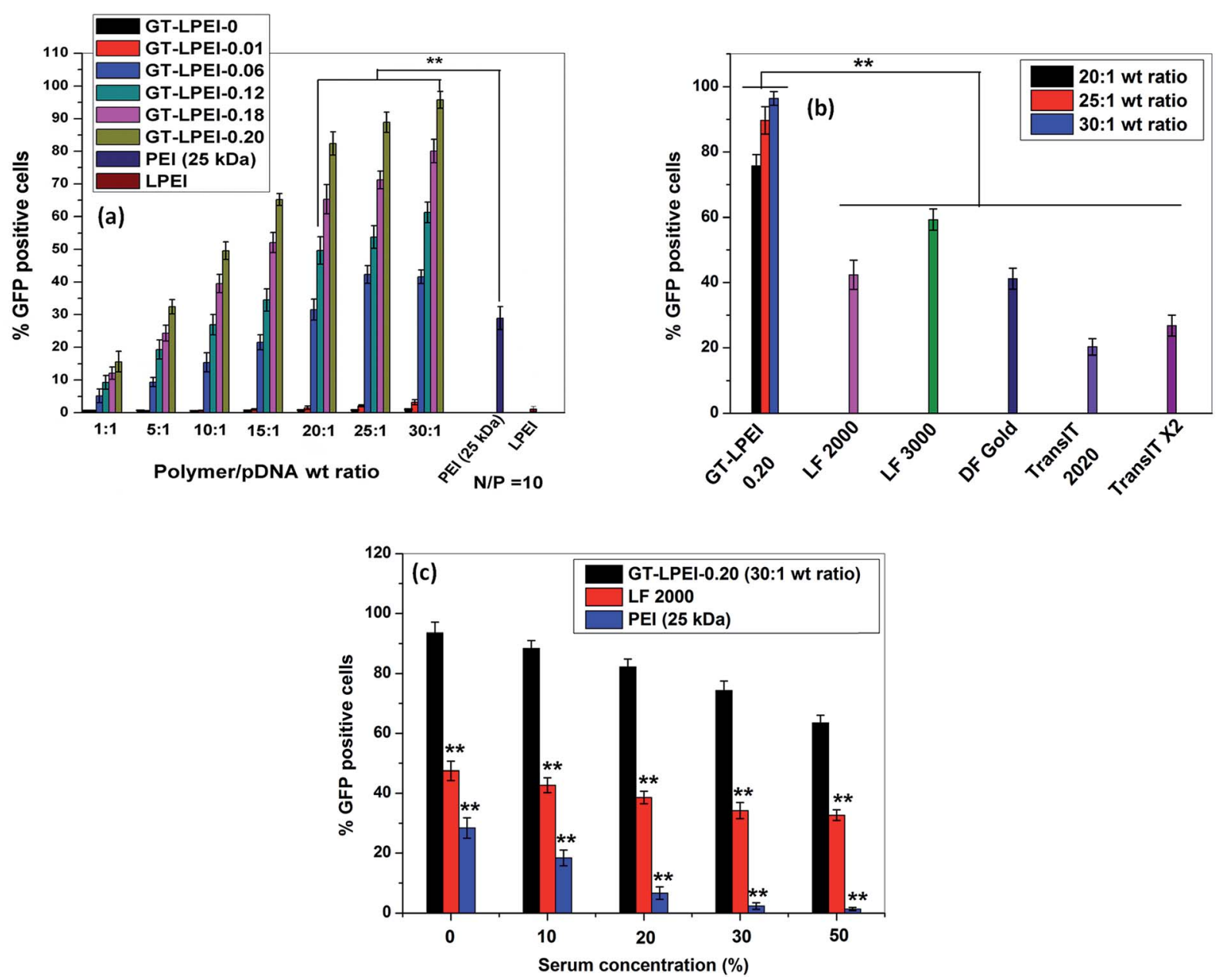

Fig. 3 (a) Flow cytometric analysis of GFP-expressing cells $48 \mathrm{~h}$ post transfection by GT/pDNA and different GT-LPEls/pDNA complexes at different weight ratios of $1: 1,5: 1,10: 1,15: 1,20: 1,25: 1$ and $30: 1, \mathrm{PEI} / \mathrm{pDNA}$ complex at N/P ratio 10 containing $1 \mu \mathrm{g}$ of pDNA' in each formulation in HeLa cells, (b) comparative transfection efficiency determined by FACS analysis of GT-LPEI-0.20/pDNA complex at weight ratios $20: 1,25: 1$ and $30: 1$ with commercialized transfection reagents and (c) effect of serum concentration on transfection efficiency of GT-LPEI$0.20 /$ pDNA complex at weight ratio of 25 : 1. LF 2000/pDNA and PEI (25 kDa)/pDNA complexes were used as positive controls. 
slightly with the increase in the polymer/DNA weight ratio but the cell viability at the highest weight ratio $(30: 1)$ was higher compared to both LF 2000/pDNA and PEI (25 kDa)/pDNA complexes in all cells.

\subsection{In vitro transfection}

Transfection efficiency of GT-LPEI was optimized in HeLa cells under different conditions. Fluorescence micrographs of cells transfected by GT/pDNA and the different GT-LPEIs/pDNA complexes at weight ratios of $20: 1,25: 1$ and $30: 1$ are shown in Fig. S7. $\uparrow$ GT/pDNA complexes at all weight ratios did not show any transfection likely due to inefficient binding with pDNA as reported above. Transfection efficiency of GT increased after conjugation of PEI with GT because of the increased DNA binding capacity. GT-LPEI-0.20/pDNA complexes showed the highest transfection efficiency at all weight ratios among all the GT-LPEIs prepared because of its superior properties in terms of size, morphology and DNA binding capability, as discussed above.

The transfection efficiency of all GT-LPEIs was further quantified by FACS analysis as shown in Fig. 3. The transfection efficiency of GT significantly increased after conjugation of PEI with GT. Transfection efficiency of all GT-LPEIs increased with the increase in weight ratio. GT-LPEI-0.20 showed highest transfection efficiency and reached up to $\sim 97 \%$ which was markedly higher than that of PEI (25 kDa)/pDNA complex (efficiency of only $\sim 30 \%$ ). The transfection efficiency of GTLPEI-0.20/pDNA complexes at weight ratios of $20: 1,25: 1$ and $30: 1$ was further compared with that of different commercially available transfection reagents including LF 2000, LF 3000, DF Gold, TransIT 2020 and TransIT X2 as shown in Fig. 3b. Strikingly, it was observed that GT-LPEI-0.20 showed superior transfection efficiency at all weight ratios compared to all

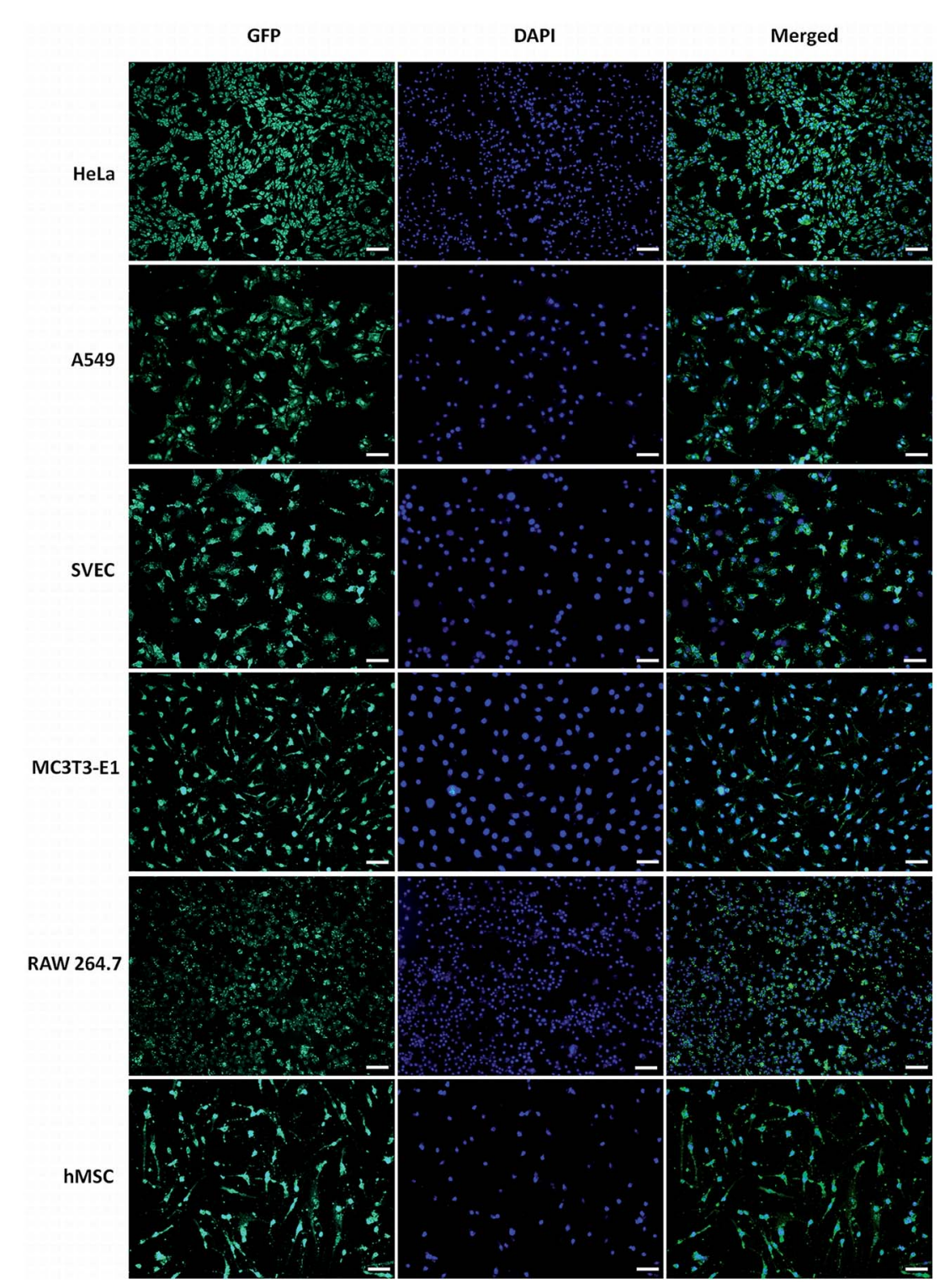

Fig. 4 Representative fluorescence micrographs of different GFP transfected and nuclear stained cells by GT-LPEI-0.20/pDNA complexes at weight ratio of $25: 1$ containing $1 \mu \mathrm{g}$ of pDNA. The scale bar is $10 \mathrm{~mm}$. 
commercialized transfection reagents. Among the commercialized reagents, LF 3000 showed highest transfection efficiency of $\sim 65 \%$ which was significantly lower compared to that seen with GT-LPEI-0.20.

The transfection efficiency of gene delivery system in presence of serum proteins is critical for successful gene therapy in vivo. Generally, it has been found that the transfection efficiency of a polymeric gene delivery system decreases sharply in the presence of serum proteins due to unwanted interactions between the protein and the gene delivery system. ${ }^{51}$ The effect of serum proteins on transfection efficiency of GT-LPEI-0.20 was assessed by varying the serum concentration from $0 \%$ to $50 \%$ as shown in Fig. 3c. The transfection efficiency of GTLPEI-0.20/pDNA complex at weight ratio of $25: 1$ decreased with the increase in serum concentration although the efficiency was $\sim 70 \%$ even at $50 \%$ serum concentration. In contrast, LF 2000 and PEI ( $25 \mathrm{kDa}$ ) yielded in only $\sim 35 \%$ and $<3 \%$ transfection, respectively at $50 \%$ serum concentration. It was found that the transfection efficiency marginally decreased in medium containing $10 \%$ serum compared to serum-free media. The particle size and zeta potential of GT-LPEI-0.20/ pDNA complexes at different weight ratio were also measured as shown in Fig. $\mathrm{S} 8 \dagger$ to assess the effect of serum proteins. The particle size increased in presence of serum containing media likely due to adsorption of negatively charged serum proteins through electrostatic interactions. Similarly, the zeta potential became negative at low weight ratio but became positive beyond weight ratio of $15: 1$ possibly because of the adsorbed proteins.

It was also observed that the morphology of transfected cells was not affected after transfection using GT-LPEI-0.20/pDNA complex at weight ratio of $25: 1$ as shown in representative micrographs for HeLa cells (Fig. S9†). GT-LPEI-0.20 alone was also used as the control during transfection. It was observed that the cells remained healthy similar to the negative control indicating that the GT-LPEI carrier is not cytotoxic even when transfection was above $90 \%$.

The transfection efficiency of GT-LPEI-0.20/pDNA complex at weight ratio of $20: 1,25: 1$ and $30: 1$ was further evaluated in various cells such as A549, SVEC, MC3T3-E1, RAW 264.7 and hMSCs. The fluorescence micrographs of all the transfected cells are shown in Fig. S10. $\dagger$ GT-LPEI-0.20/pDNA complexes at all weight ratios showed discernibly higher transfection compared to LF 2000 in all the cell lines and the primary human stem cells. To visually demonstrate the high transfection efficiency of GT-LPEI/pDNA complex, the nuclei of the all cells were stained by DAPI and overlaid as merged micrographs as shown in Fig. 4. Note that nearly all the cells in the different cell types were indeed transfected as there was good overlap between GFP expression and nuclear staining. The cells appeared healthy and well spread with no apparent signs of cytotoxicity. The transfection efficiency was further quantified by FACS analysis to validate the fluorescence and staining data. Fig. 5a shows the transfection efficiency of GT-LPEI-0.20/pDNA complex at weight ratios of $20: 1,25: 1$ and $30: 1$ quantified by FACS in the various cells. The transfection efficiency of GTLPEI-0.20 at all weight ratios was significantly higher compared to that of LF 2000 and PEI (25 kDa). The exceptionally high transfection efficiency of GT-LPEI-0.20 may be attributed to its ability to large DNA binding capacity by self assembly, cylindrical morphology and the large availability of cell-adhesive peptide sequences such as RGD in the gelatin which likely augmented cellular uptake and intracellular transport.

\subsection{Intracellular kinetics}

Intracellular uptake mechanism is another important parameter that determines gene transfection efficiency along with other parameters such as DNA complexation capability, particle size, morphology and zeta potential. ${ }^{52,53}$ Clathrin, caveolae and macropinocytosis mediated endocytosis pathways are the major uptake pathways for cellular internalization of a polymer/DNA complex. $^{54}$ To understand the uptake mechanism, the
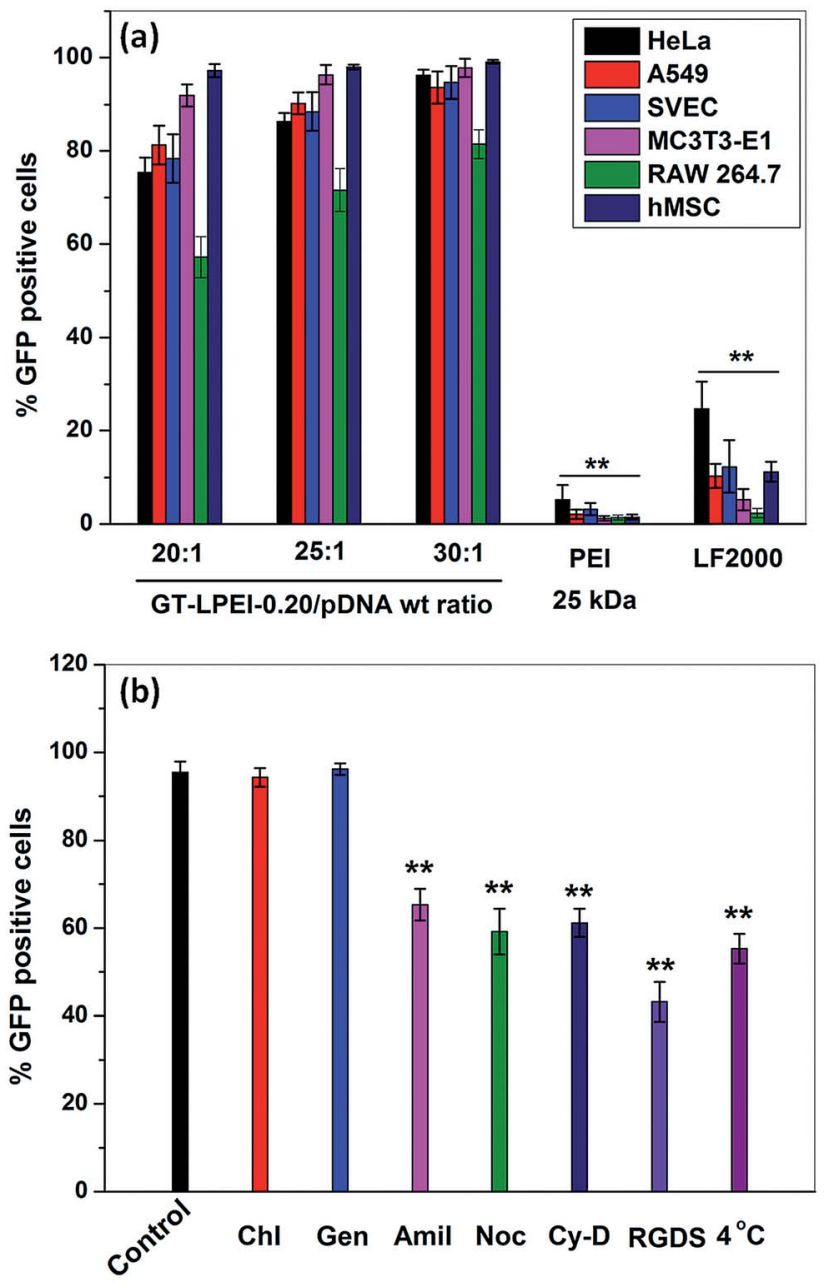

Fig. 5 (a) Flow cytometric analysis of GFP-expressing cells $48 \mathrm{~h}$ post transfection by GT/pDNA and GT-LPEI-0.20/pDNA complexes at different weight ratios of $20: 1,25: 1$ and $30: 1$, PEI/pDNA complex at N/P ratio 10 and LF 2000/pDNA complex containing $1 \mu \mathrm{g}$ of pDNA in each formulation against various cells and (b) relative transfection efficiency determined by FACS analysis of GT-LPEI-0.20/pDNA complex at the weight ratio of $25: 1$ against HeLa cell after treating with various endocytotic inhibitors for $1 \mathrm{~h}$ before transfection containing $1 \mu \mathrm{g}$ of pDNA followed by further incubation for $48 \mathrm{~h}$. 
transfection efficiency of GT-LPEI-0.20/pDNA complex at weight ratio of $25: 1$ was assessed in HeLa cells in the presence of different drugs, peptides and low temperature conditions such as chlorpromazine, genistein, amiloride, nocodazole, cytochalasin D, exogenous soluble RGDS peptide and $4{ }^{\circ} \mathrm{C}$ temperature. Fig. 5b shows that low temperature significantly decreased the transfection efficiency of GT-LPEI-0.20 indicating that the cellular uptake of GT-LPEI-0.20/pDNA complex occurred through energy dependent endocytosis pathway. The transfection efficiency was not affected by chlorpromazine and genistein demonstrating that clathrin and caveolae mediated endocytosis pathway were not involved in the cellular uptake of the GT-LPEI-0.20/pDNA complex. In contrast, amiloride significantly inhibited the transfection efficiency $(\sim 32 \%$ inhibition) indicating that macropinocytosis mediated endocytosis was the primary uptake mechanism of the GT-LPEI-0.20/pDNA complex. A previous study reported that the clathrin mediated endocytosis pathway is primarily involved for particles of size $<200 \mathrm{~nm}$ but caveolae and macro-pinocytosis mediated endocytosis are the primary pathways for particles $>200 \mathrm{~nm} \cdot{ }^{55,56} \mathrm{In}$ this study, the particle size of GT-LPEI-0.20/pDNA complex was around $250 \mathrm{~nm}$ which are in good agreement with the previous report. Khalil et $a .^{57}$ reported that the macropinocytosis mediated endocytosis pathway is more favorable for gene transfection efficiency than clathrin endocytosis because macropinocytosis does not involve degradative lysosome compartment during internalization.

The transgene expression is not only dependent on cellular uptake pathway but also depends on the rate of endosomal release inside the cell. ${ }^{58}$ El-Sayed et $a l .{ }^{59}$ reported the effect of surface modification of nanoparticle by octaarginine (R8) and octalysine (K8) on endosomal escape rate and consequently the transfection efficiency. Both R8 and K8 modified nanoparticles entered into the cell through macropinocytosis mediated pathway but R8 modified nanoparticle showed better transfection efficiency. They explained that R8 modified nanoparticle showed higher escape rate from endosome due to the positive charge. In this report, GT-LPEI-0.20/pDNA complex with positive zeta potential at weight ratio of $25: 1$ also followed the macropinocytosis mediated endocytosis pathway. Therefore, GT-LPEI-0.20/pDNA complex might have higher endosomal escape rate resulting in high transfection efficiency. It is also observed from Fig. 5b, the transfection efficiency was also inhibited by nocodazole and cytochalasin $\mathrm{D}$ demonstrating that the nuclear transport of GT-LPEI-0.20/pDNA complex occurred through actin and microtubule. Previous reports suggest that

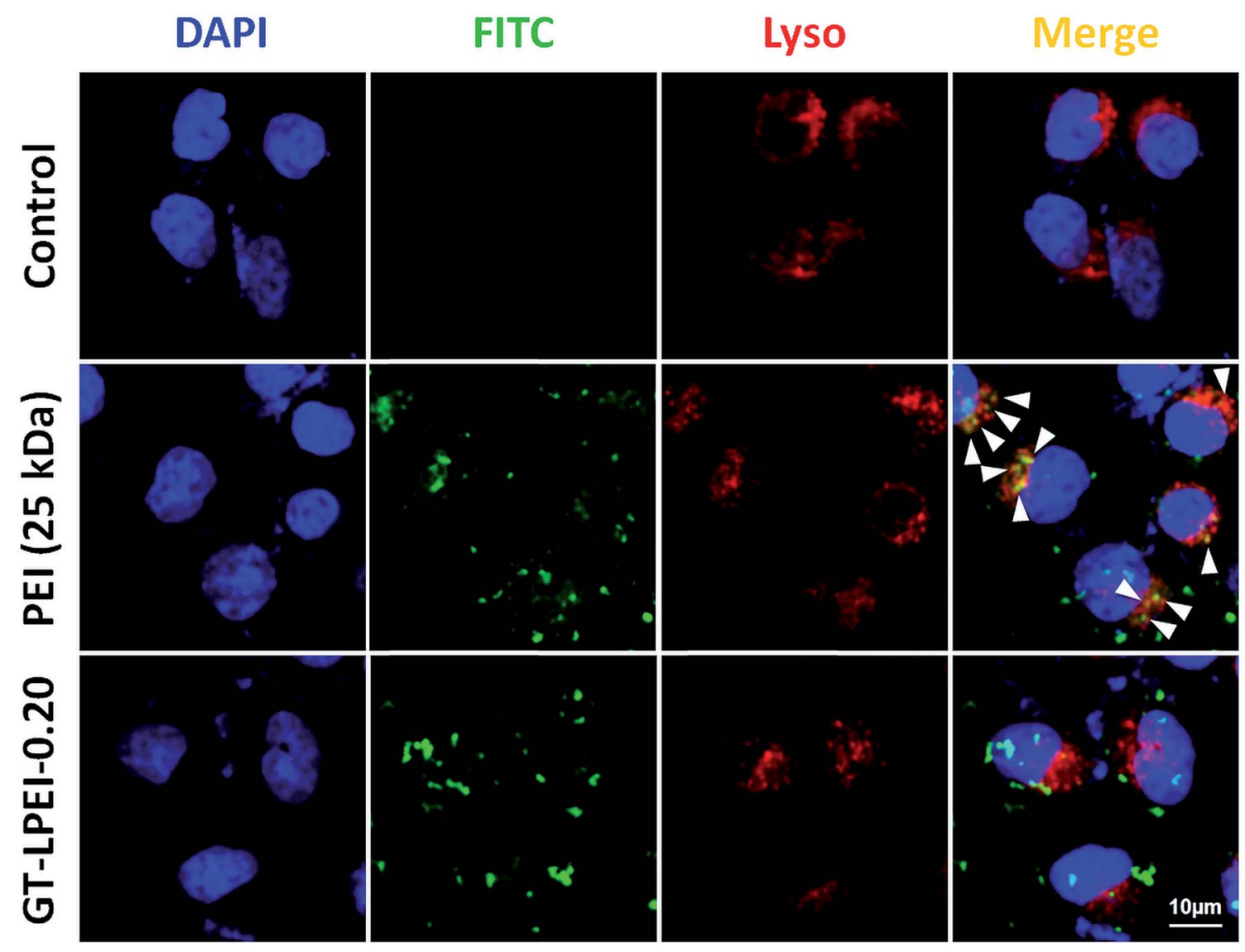

Fig. 6 CLSM images of Lysotracker colocalization assay in HeLa cells after $4 \mathrm{~h}$ of transfection with FITC-labelled PEI/pDNA complex at N/P ratio of 10 and GT-LPEI-0.20/pDNA complex at weight ratio of $25: 1$ containing $1 \mu \mathrm{g}$ pDNA in each formulation. For each panel, images from left to right show stained cell nuclei (blue), FITC labelled polymer (green), Lysotracker (red) and colocalization of green and red in merged (yellow). Scale bar is $10 \mu \mathrm{m}$. 
the nuclear internalization of polymer/DNA through actin and microtubule enhances the transfection efficiency. ${ }^{\mathbf{6 0 , 6 1}}$ The transfection efficiency was also reduced by the presence of exogenous RGDS peptide indicating that integrin mediated cellular uptake is involved during uptake of the complex.

\subsection{Intracellular distribution and kinetics}

Intracellular distribution of polymer/DNA complex after cellular uptake is an important parameter for efficient transgene expression. After cellular uptake, the polymer/DNA complex has to overcome several obstacles in the form of transport and degradative mechanisms within the cell. To understand the intracellular distribution kinetics of GT-LPEI-0.20/pDNA complex at weight ratio of $25: 1$ using Cy3 labeled pDNA, the CLSM images were captured at different cellular uptake time points $(0,0.5,2$ and $4 \mathrm{~h})$ as shown in Fig. S11. $\dagger$ It is observed that a few complexes (red dots) entered into the cell $0.5 \mathrm{~h}$ post transfection and the number of red dots (complexes) increased with time. Few complexes reached the nucleus within $2 \mathrm{~h}$ post transfection and the number of complexes at the nucleus further increased at $4 \mathrm{~h}$ demonstrating rapid nuclear transport of the complexes which facilitates high transfection efficiency.

The intracellular distribution of GT-LPEI-0.20/pDNA complex at weight ratio of $25: 1$ was also compared to PEI (25 $\mathrm{kDa} / \mathrm{pDNA}$ and LF 2000/pDNA complexes as shown in Fig. S12. $\dagger$ pDNA alone was used as the negative control. It can be seen that few PEI $(25 \mathrm{kDa}) /$ pDNA complexes entered into the cell after $4 \mathrm{~h}$ post transfection and most of the complexes were located away from the nucleus suggesting poor intracellular transport leading to low transfection efficiency of PEI ( $25 \mathrm{kDa})$. In contrast, the number of complexes associated with both LF 2000/pDNA and GT-LPEI-0.20/pDNA complex was higher but GT-LPEI-0.20/pDNA complexes showed fastest transport and augmented transfection efficiency.

Cellular internalization after uptake is a major internal barrier in nonviral gene delivery systems. ${ }^{62}$ It has been reported that most nonviral vectors enter cells through clathrin mediated endocytosis pathway followed by internalization in to degradative lysosomal compartment resulting in low transfection efficiency ${ }^{63}$ Lysotracker colocalization assay was performed to assess the involvement of lysosomal compartment in the uptake and intracellular transport of the GT-LPEI carrier (Fig. 6). It was observed that most of the PEI (green) colocalized with the lysosomes (red) as indicated by the arrows (yellow spots) demonstrating that PEI/pDNA complexes are entrapped in the lysosomal degradative compartment resulting in lower transfection efficiency. In contrast, the GT-LPEI-0.20/pDNA complex escaped the lysosomal compartment (minimal colocalization) that likely results in higher transfection efficiency.

\subsection{In vivo transfection}

For clinical gene therapy application, the gene delivery system should be able to delivery therapeutic gene efficiently and safely in vivo. Toward development for clinical use, a preliminary experiment was performed in mice to assess the efficiency of GT-LPEI-0.20/pDNA complex injected intraperitoneally. pDNA

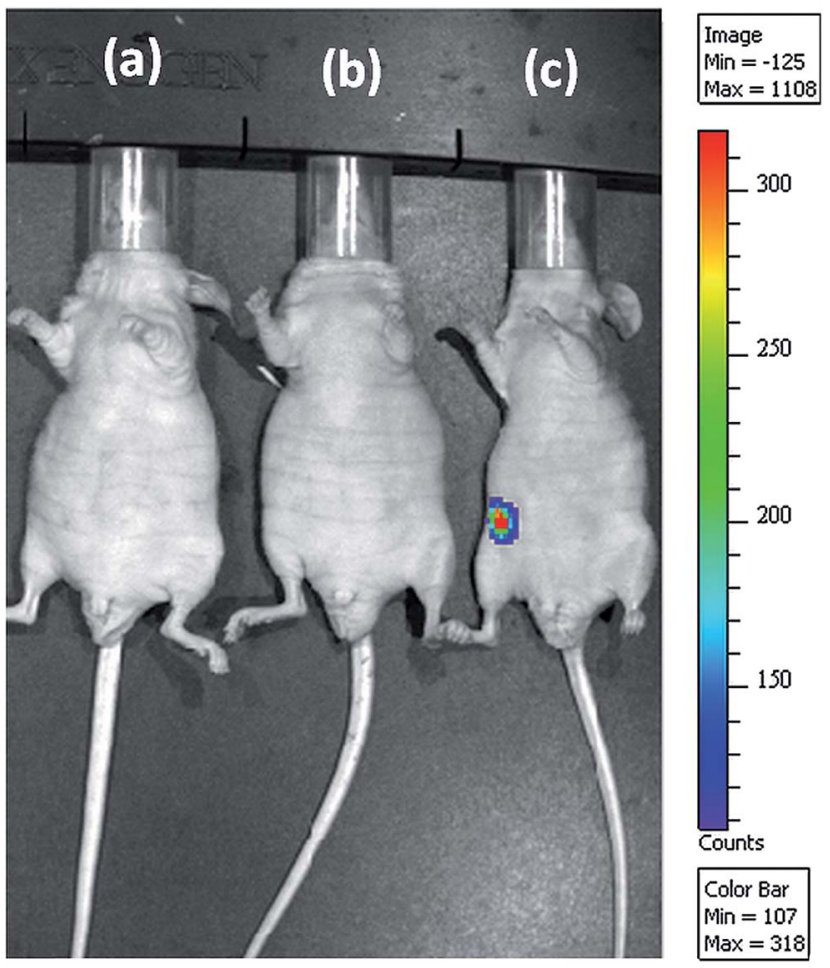

Fig. 7 Representative bioluminescence live animal imaging of gene expression $24 \mathrm{~h}$ after intraperitoneal injection of (a) luciferaseencoding pDNA only, (b) PEl ( $25 \mathrm{kDa}) / \mathrm{pDNA}$ complex at N/P ratio of 10 and (c) GT-LPEI-0.20/pDNA complex at weight ratio of $25: 1$ containing $40 \mu \mathrm{g}$ pDNA in each formulation. The scale indicates surface radiance (photons per $\mathrm{s}$ per $\mathrm{cm}^{2}$ per steradian).

alone and PEI (25 kDa)/pDNA complexes were used as the negative and positive controls, respectively. The luciferase expression was visualized $24 \mathrm{~h}$ post injection as shown in representative images in Fig. 7. Strong signal from the peritoneum of the animal injected with GT-LPEI-0.20/pDNA complex indicating the GT-LPEI- 0.20 is able to transfect in vivo. In contrast, pDNA and PEI $(25 \mathrm{kDa}) /$ pDNA did not show any measurable gene expression also supports the in vitro data. Therefore, the novel GT-LPEI complex developed herein is a promising nonviral carrier for gene therapy that may be further developed for clinical use.

\section{Conclusion}

LPEI was conjugated with gelatin through naphthalimide moiety to avoid self-crosslinking of gelatin to yield GT-LPEI. The LPEI content was optimized to yield GT-LPEI- 0.20 with good pDNA complexation ability. Self-assembly of GT-LPEI-0.20 with pDNA yielded cylindrical nanoparticles of $\sim 250 \mathrm{~nm}$ in size with zeta potential of $\sim 27 \mathrm{mV}$. GT-LPEI-0.20 showed exceptionally high transfection in a wide variety of mammalian cells surpassing the efficiency offered by many different commercially available transfection reagents. GT-LPEI uptake is shown to be mediated by macro-pinocytosis followed by rapid transport to the nucleus facilitated by F-actin with microtubules 
without entrapment in the lysosomal compartment. The efficacy of GT-LPEI system in vivo was also demonstrated. Therefore, GT-LPEI is a promising nonviral carrier for gene therapy.

\section{Financial disclosure}

Patent application has been filed.

\section{Acknowledgements}

This work was funded by the Nanomission Program of the Department of Science and Technology (DST), India. K. S. was supported by the D. S. Kothari fellowship (BSR/EN/13-14/0005) from the University Grants Commission (UGC), India. K. C. acknowledges the Ramanujan fellowship from DST. The authors thank AFMM, the NMR Center and the Central Confocal Imaging Facility for access to equipments.

\section{References}

1 H. Yin, R. L. Kanasty, A. A. Eltoukhy, A. J. Vegas, J. R. Dorkin and D. G. Anderson, Nat. Rev. Genet., 2014, 15, 541-555.

2 D. W. Pack, A. S. Hoffman, S. Pun and P. S. Stayton, Nat. Rev. Drug Discovery, 2005, 4, 581-593.

3 M. Al-Dosari and X. Gao, AAPS J., 2009, 11, 671-681.

4 P. P. Kundu and K. Sarkar, in Biopolymers: Biomedical and Environmental Applications, ed. S. Kalia and L. Avérous, John Wiley \& Sons, Inc., 2011, DOI: 10.1002/ 9781118164792.ch20, pp. 575-603.

5 X. Guo and L. Huang, Acc. Chem. Res., 2011, 45, 971-979.

6 K. Ewert, A. Zidovska, A. Ahmad, N. Bouxsein, H. Evans, C. McAllister, C. Samuel and C. Safinya, in Nucleic Acid Transfection, ed. W. Bielke and C. Erbacher, Springer, Berlin Heidelberg, 2010, vol. 296, ch. 70, pp. 191-226.

7 S. C. de Smedt, J. Demeester and W. E. Hennink, Pharm. Res., 2000, 17, 113-126.

8 K. Sarkar, R. Srivastava, U. Chatterji and P. Kundu, J. Appl. Polym. Sci., 2011, 121, 2239-2249.

9 U. Lungwitz, M. Breunig, T. Blunk and A. Göpferich, Eur. J. Pharm. Biopharm., 2005, 60, 247-266.

10 M. Thomas and A. M. Klibanov, Proc. Natl. Acad. Sci. U. S. A., 2002, 99, 14640-14645.

11 K. Kunath, A. von Harpe, D. Fischer, H. Petersen, U. Bickel, K. Voigt and T. Kissel, J. Controlled Release, 2003, 89, 113125.

12 C. H. Jones, C.-K. Chen, A. Ravikrishnan, S. Rane and B. A. Pfeifer, Mol. Pharmaceutics, 2013, 10, 4082-4098.

13 D. Lechardeur and G. L. Lukacs, Curr. Gene Ther., 2002, 2, 183-194.

14 K. Kunath, T. Merdan, O. Hegener, H. Häberlein and T. Kissel, J. Gene Med., 2003, 5, 588-599.

15 S. Mahor, B. C. Dash, S. O'Connor and A. Pandit, Bioconjugate Chem., 2012, 23, 1138-1148.

16 T. Merdan, K. Kunath, H. Petersen, U. Bakowsky, K. H. Voigt, J. Kopecek and T. Kissel, Bioconjugate Chem., 2005, 16, 785792.
17 V. B. Morris, C. K. S. Pillai and C. P. Sharma, Polym. Int., 2011, 60, 1097-1106.

18 F. Kratz, J. Controlled Release, 2008, 132, 171-183.

19 M. Bachar, A. Mandelbaum, I. Portnaya, H. Perlstein, S. Even-Chen, Y. Barenholz and D. Danino, J. Controlled Release, 2012, 160, 164-171.

20 J. Kundu, Y.-I. Chung, Y. H. Kim, G. Tae and S. Kundu, Int. J. Pharm., 2010, 388, 242-250.

21 N. Saranya, A. Moorthi, S. Saravanan, M. P. Devi and N. Selvamurugan, Int. J. Biol. Macromol., 2011, 48, 234-238.

22 A. O. Elzoghby, W. M. Samy and N. A. Elgindy, J. Controlled Release, 2012, 161, 38-49.

23 P. Kozlov and G. Burdygina, Polymer, 1983, 24, 651-666.

24 H. Wang, O. C. Boerman, K. Sariibrahimoglu, Y. Li, J. A. Jansen and S. C. Leeuwenburgh, Biomaterials, 2012, 33, 8695-8703.

25 M. Nahar, D. Mishra, V. Dubey and N. K. Jain, Nanomedicine: Nanotechnology, Biology and Medicine, 2008, 4, 252-261.

26 E. Leo, M. A. Vandelli, R. Cameroni and F. Forni, Int. J. Pharm., 1997, 155, 75-82.

27 Y.-Z. Zhao, X. Li, C.-T. Lu, Y.-Y. Xu, H.-F. Lv, D.-D. Dai, L. Zhang, C.-Z. Sun, W. Yang and X.-K. Li, Acta Diabetol., 2012, 49, 315-325.

28 R. Kumar, R. C. Nagarwal, M. Dhanawat and J. K. Pandit, J. Biomed. Nanotechnol., 2011, 7, 325-333.

29 S. K. Jain, Y. Gupta, A. Jain, A. R. Saxena, P. Khare and A. Jain, Nanomedicine: Nanotechnology, Biology and Medicine, 2008, 4, 41-48.

30 G. Kaul and M. Amiji, J. Pharm. Sci., 2005, 94, 184-198.

31 M. D. Bhavsar and M. M. Amiji, AAPS PharmSciTech, 2008, 9, 288-294.

32 M. Bhavsar and M. Amiji, Gene Ther., 2008, 15, 1200-1209.

33 G. K. Zorzi, J. E. Párraga, B. Seijo and A. Sánchez, Macromol. Biosci., 2011, 11, 905-913.

34 X.-H. Tian, Z.-G. Wang, H. Meng, Y.-H. Wang, W. Feng, F. Wei, Z.-C. Huang, X.-N. Lin and L. Ren, Int. J. Nanomed., 2013, 8, 865.

35 K. Sarkar, M. Debnath and P. Kundu, Carbohydr. Polym., 2013, 92, 2048-2057.

36 C. N. Grover, J. H. Gwynne, N. Pugh, S. Hamaia, R. W. Farndale, S. M. Best and R. E. Cameron, Acta Biomater., 2012, 8, 3080-3090.

37 K. Sarkar, A. Chatterjee, G. Chakraborti and P. P. Kundu, Carbohydr. Polym., 2013, 98, 596-606.

38 K. Sarkar, S. L. Banerjee, P. P. Kundu, G. Madras and K. Chatterjee, J. Mater. Chem. B, 2015, 3, 5266-5276.

39 Q. Hu, J. Wang, J. Shen, M. Liu, X. Jin, G. Tang and P. K. Chu, Biomaterials, 2012, 33, 1135-1145.

40 X. Hu, D. Li, F. Zhou and C. Gao, Acta Biomater., 2011, 7, 1618-1626.

41 K. Sarkar and P. P. Kundu, Int. J. Biol. Macromol., 2012, 51, 859-867.

42 R. I. Mahato and S. W. Kim, Pharmaceutical Perspectives of Nucleic Acid-Based Therapy, CRC Press, 2003.

43 K. Sarkar and P. Kundu, Carbohydr. Polym., 2013, 98, 495504. 
44 X. Zhao, Z. Li, H. Pan, W. Liu, M. Lv, F. Leung and W. W. Lu, Acta Biomater., 2013, 9, 6694-6703.

45 B. Lu, D.-Q. Wu, H. Zheng, C.-Y. Quan, X.-Z. Zhang and R.-X. Zhuo, Mol. BioSyst., 2010, 6, 2529-2538.

46 J. Shi, J. L. Choi, B. Chou, R. N. Johnson, J. G. Schellinger and S. H. Pun, ACS Nano, 2013, 7, 10612-10620.

47 S. E. Gratton, P. A. Ropp, P. D. Pohlhaus, J. C. Luft, V. J. Madden, M. E. Napier and J. M. DeSimone, Proc. Natl. Acad. Sci. U. S. A., 2008, 105, 11613-11618.

48 B. Sternberg, K. Hong, W. Zheng and D. Papahadjopoulos, Biochim. Biophys. Acta Biomembr., 1998, 1375, 23-35.

49 B. Gaihre, M. S. Khil and H. Y. Kim, J. Microencapsulation, 2011, 28, 286-293.

50 S. Hong, P. R. Leroueil, E. K. Janus, J. L. Peters, M.-M. Kober, M. T. Islam, B. G. Orr, J. R. Baker and M. M. Banaszak Holl, Bioconjugate Chem., 2006, 17, 728-734.

51 P. P. Kundu and V. Sharma, Curr. Opin. Solid State Mater. Sci., 2008, 12, 89-102.

52 M. van der Aa, U. Huth, S. Häfele, R. Schubert, R. Oosting, E. Mastrobattista, W. Hennink, R. Peschka-Süss, G. Koning and D. Crommelin, Pharm. Res., 2007, 24, 1590-1598.
53 C. W. Pouton and L. W. Seymour, Adv. Drug Delivery Rev., 1998, 34, 3-19.

54 A. F. Adler and K. W. Leong, Nano Today, 2010, 5, 553-569.

55 J. Rejman, V. Oberle, I. Zuhorn and D. Hoekstra, Biochem. J., 2004, 377, 159-169.

56 A. El-Sayed and H. Harashima, Mol. Ther., 2013, 21, 11181130.

57 I. A. Khalil, K. Kogure, S. Futaki and H. Harashima, J. Biol. Chem., 2006, 281, 3544-3551.

58 M. Magzoub, A. Pramanik and A. Gräslund, Biochemistry, 2005, 44, 14890-14897.

59 A. El-Sayed, I. A. Khalil, K. Kogure, S. Futaki and H. Harashima, J. Biol. Chem., 2008, 283, 23450-23461.

60 K. Tanaka, T. Kanazawa, K. Sugawara, S. Horiuchi, Y. Takashima and H. Okada, Int. J. Pharm., 2011, 419, 231234.

61 L. Pigeon, C. Gonçalves, D. Gosset, C. Pichon and P. Midoux, Small, 2013, 9, 3845-3851.

62 D. Cross and J. K. Burmester, Clin. Med. Res., 2006, 4, 218227.

63 R. Wattiaux, N. Laurent, S. Wattiaux-de Coninck and M. Jadot, Adv. Drug Delivery Rev., 2000, 41, 201-208. 